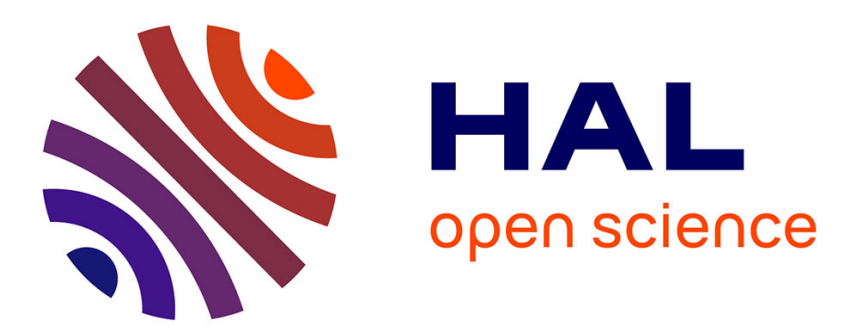

\title{
Linearly polarized laser beam with generalized boundary condition and non-paraxial corrections
}

Huan Wang, Loïc Amoudry, Kevin Cassou, Kevin Dupraz, Wenhui Huang, Aurélien Martens, Daniele Nutarelli, Chuanxiang Tang, Lixin Yan, Fabian Zomer

\section{To cite this version:}

Huan Wang, Loïc Amoudry, Kevin Cassou, Kevin Dupraz, Wenhui Huang, et al.. Linearly polarized laser beam with generalized boundary condition and non-paraxial corrections. J.Opt.Soc.Am.A, 2019, 36 (12), pp.1949. 10.1364/josaa.36.001949 . hal-02394733

\section{HAL Id: hal-02394733 \\ https://hal.science/hal-02394733}

Submitted on 26 Nov 2020

HAL is a multi-disciplinary open access archive for the deposit and dissemination of scientific research documents, whether they are published or not. The documents may come from teaching and research institutions in France or abroad, or from public or private research centers.
L'archive ouverte pluridisciplinaire HAL, est destinée au dépôt et à la diffusion de documents scientifiques de niveau recherche, publiés ou non, émanant des établissements d'enseignement et de recherche français ou étrangers, des laboratoires publics ou privés. 


\title{
Linearly polarized laser beam with generalized boundary condition and non-paraxial corrections
}

\author{
Huan Wang ${ }^{1,2,}$, Loïc Amoudry ${ }^{1}$, Kevin Cassou ${ }^{1}$, Kevin Dupraz ${ }^{1}$, Wenhui Huang ${ }^{2}$, Aurélien \\ Martens $^{1}$, Daniele NutArelli ${ }^{1}$, ChuanXiang TANG ${ }^{2}$, Lixin YAn $^{2}$, And Fabian Zomer ${ }^{1}$ \\ ${ }^{1}$ LAL, Univ. Paris-Sud, CNRS/IN2P3, Université Paris-Saclay, Orsay, France \\ ${ }^{2}$ Key Laboratory of Particle and Radiation Imaging (Ministry of Education) and Department of Engineering Physics, Tsinghua University, Beijing 10084, China \\ *Corresponding author: huanwanghw@foxmail.com
}

Compiled November 18, 2020

\begin{abstract}
Monochromatic linearly polarized Gaussian beams tightly focused by a perfect parabola modeled with the integral formalism of Ignatovsky are found to be well approximated with a generalized Lax series expansion beyond the paraxial approximation. This allows to obtain simple analytic formulae of the electromagnetic field both in the direct and momentum spaces. It significantly reduces the computing time, especially when dealing with the problem of simulating direct laser acceleration. The series expansion formulation depends on integration constants that are linked to boundary conditions. They were found to significantly depend on the region of space over which the integral formulation is fit. Consequently the net acceleration of electrons initially at rest is extremely sensitive to the chosen set of initial parameters due to the extreme focusing investigated here. This suggests to avoid too tight focusing schemes in order to obtain reliable predictions when the process of interest is mainly sensitive to the field and not the intensity. () 2020 Optical Society of America
\end{abstract}

http://dx.doi.org/10.1364/ao.XX.XXXXXX

\section{INTRODUCTION}

With the advances in laser technology in the last decades, direct acceleration of particles in vacuum by lasers (VLA) has been achieved. Acceleration of about $25 \mathrm{keV}$ electrons initially at rest in a gas cell was obtained in 2012 [1]. This technology may thus enable compact, low-energy and ultra-short electron beams of interest for electron diffraction experiment [2], that is difficult to obtain with conventional radiofrequency accelerators. Alternatively electron beams carrying higher charges with several $\mathrm{MeV}$ of energy were obtained by creation of plasma mirror from which electrons are extracted and accelerated [3]. Thus applications as all-optical X-ray sources, already demonstrated with plasma cells [4], may be envisaged by means of Compton back-scattering of an intense laser on $\mathrm{MeV}$ range electron beams obtained by VLA.

However the very large oscillation frequency of the optical field and the requested tight focusing of the laser beam makes this technology highly sensitive to jitters, misalignments and departure from perfect focusing of the laser beam. Performance of the acceleration process has been shown to be extremely sensitive to the choice of a set of initial parameters [5]. Thus a description of the laser field in the focusing region beyond the paraxial approximation is often needed. To this end several approaches have been used in the past to model the laser beam in the focus- ing region, each corresponding to different assumptions. Closed form solutions satisfying exactly Maxwell equations were obtained for radially polarized laser beams provide self-consistent expressions that however correspond to definite boundary conditions that may not be representative of an actual experiment [6]. Alternatively, Maxwell equations consistent integral expressions are also available but suffer from approximations and are also bound to specific boundary conditions [7]. Finally, the historical technique consisting in expanding paraxial solutions of the wave equations in series [8] has been recently shown to offer the unique ability to account for arbitrary boundary conditions [9]. This ability may be seen as crucial when considering the sensitivity of the acceleration process to boundary conditions that is likely to limit the predictive power of detailed and expansive simulations.

Some recent research activity has concentrated on introducing temporal effects beyond the slowly envelope approximation. These effects are indeed of importance, especially when dealing with longitudinal chromatism [10]. Such spatio-temporal couplings can also be included within perturbation series expansions [11] for few cycle fields. Solving the Maxwell equations in a consistent way at a given order of the series expansion in momentum space including such effects was demonstrated to match numerical calculations with good precision [12], though requiring numerical integration to obtain position space field 
values.

Though the vectorial approach is elegant and provide selfconsistent solutions to the Maxwell equations, but still suffers from some approximations, the resulting integral expressions require large computational time, especially when precision is required, which may not be practical in many cases. Also they are usually bound to given boundary conditions that may not be relevant to a specific experimental situation [9]. The sensitivity to these may thus not be easy to gauge, being a critical aspect for experimental demonstration. An alternative approach consist in a generalized series expansion [9] which is self-consistent only at a given order of the series expansion (this may not be of numerical importance) but allows to assert the sensitivity to a choice of boundary conditions (i.e. non-predictive aspects of a specific experiment thus giving a clue for tolerance studies) and analytic direct-space expressions, that are computationally efficient. As mentioned in Ref. [7], this approach entails far-field divergences [13] that are sometimes of importance in the phase space of interest for the studied problem, though this problem can be solved[14].

It is sometimes thought that vectorial models cannot be represented by means of series expansions [7] whereas providing more accurate expressions of the fields. The first goal of this paper is to question these statements. Secondly we intend to apply both approaches to the particular application of direct laser acceleration. For this purpose we consider a simplified geometry that is difficult to experimentally implement, but still allows to draw conclusions, that will impact further studies on this topic. A generalized perturbation expansion method is applied to linearly polarized laser beam and compared with the Ignatovsky solution [15] revisited in Ref. [7]. The formalism of the former (latter) is presented in section 2 (3). Coefficients of the series expansion are fit to the expressions of Ignatovsky in section 4, and further applied to the specific case of direct electron acceleration in section 5 .

\section{SERIES EXPANSION FORMALISM}

Linearly polarized electromagnetic field expressions beyond paraxial approximation can be obtained by means of series expansion [8] with the prescription of Davis [16]. Though a careful explicit symmetrization of the result is required [17] which is naturally embedded in the Hertz potentials $\vec{\Pi}_{e}, \vec{\Pi}_{m}[18,19]$. The electromagnetic fields read

$$
\begin{aligned}
& \vec{E}=\vec{\nabla} \times \vec{\nabla} \times \vec{\Pi}_{e}-\mu_{0} \frac{\partial}{\partial t} \vec{\nabla} \times \vec{\Pi}_{m} \\
& \vec{B}=\mu_{0} \vec{\nabla} \times \vec{\nabla} \times \vec{\Pi}_{m}+\frac{1}{c^{2}} \frac{\partial}{\partial t} \vec{\nabla} \times \vec{\Pi}_{e},
\end{aligned}
$$

in which, for a linearly polarized wave focused along $\mathrm{z}$ axis and polarized along $x$ axis, $\vec{\Pi}_{e}=\hat{a}_{x} \Psi(x, y, z, t), \vec{\Pi}_{m}=$ $\hat{a}_{y} \eta_{0}^{-1} \Psi(x, y, z, t)$, where $\hat{a}_{x, y, z}$ are unit vectors in the directions $x, y, z$ of the reference frame. It is explicit in these expressions that a single scalar function $\Psi(x, y, z, t)$ is required to describe the focused field. Scalar and vector potentials are then related to the Hertz potentials by $V \equiv-\vec{\nabla} \cdot \vec{\Pi}_{e}$ and $\vec{A} \equiv\left(1 / c^{2}\right) \partial \vec{\Pi}_{e} / \partial t+\mu_{0} \vec{\nabla} \times \vec{\Pi}_{m}$, since $\vec{B} \equiv \vec{\nabla} \times \vec{A}$ and $\vec{E} \equiv$ $-\vec{\nabla} V-\partial \vec{A} / \partial t$ in the Lorenz gauge. It is thus possible to expand perturbatively the scalar function $\Psi(x, y, z, t)$ in series to derive field expressions beyond the paraxial approximation, but it is rather usually performed with the scalar function $\mathcal{A}(x, y, z, t)$ that defines the vector potential $\vec{A}^{\prime}(x, y, z, t)=\hat{a_{x}} \mathcal{A}(x, y, z, t)$ and its symmetric counterpart $\vec{A}^{\prime \prime}(x, y, z, t)=\hat{a_{y}} \mathcal{A}(x, y, z, t)$, that allows to rewrite the electromagnetic fields $\vec{E}=\vec{E}^{\prime}+\vec{E}^{\prime \prime}$ and $\vec{B}=\vec{B}^{\prime}+\vec{B}^{\prime \prime}$ where $\vec{B}^{\prime}=\vec{\nabla} \times \vec{A}^{\prime}$ and $\vec{E}^{\prime \prime}=\vec{\nabla} \times \vec{A}^{\prime \prime}$. The $\vec{E}^{\prime}$ and $\vec{B}^{\prime \prime}$ are consistently defined by the Maxwell equations. Both approaches provide equivalent fields at first order in the expansion series. The expansion could also be performed on the six fields projections themselves [12], which provides another equivalent procedure at first order in the expansion. In the following, we choose to follow the customary procedure that consists in expanding the scalar function $\mathcal{A}(x, y, z, t)$ by writing it in the form

$$
\mathcal{A}(x, y, z, t)=\mathcal{A}_{0} g\left(\phi / \phi_{0}\right) e^{i(\phi-\delta \phi)} \psi(x, y, z),
$$

where $g\left(\phi / \phi_{0}\right)$ is the envelope of the pulse, $\delta \phi$ the carrier to envelope phase shift, $\phi=k z-\omega_{0} t, \phi_{0}=\omega_{0} \tau / g^{-1}(\exp (-1)), \tau$ is the single-sided $\exp (-1)$ pulse width and $\psi(x, y, z)$ is the spatial profile. The scalar wave equation $\nabla^{2} \mathcal{A}-\frac{1}{c^{2}} \frac{\partial^{2}}{\partial t^{2}} \mathcal{A}=$ 0 simplifies under slowly varying envelope approximation, $\left|\frac{1}{g\left(\phi / \phi_{0}\right)} \frac{\partial g\left(\phi / \phi_{0}\right)}{\partial \phi}\right| \ll 1$, into a scalar equation for the spatial envelope $\nabla^{2} \psi+2 i k \frac{\partial \psi}{\partial z}=0$. Employing dimensionless coordinates, $\vec{\rho}=(\xi, \eta)=(x, y) / w_{0}$ and $\zeta=z / z_{R}$, it reads $\nabla_{\perp}^{2} \psi+\varepsilon^{2} \frac{\partial^{2}}{\partial \zeta^{2}} \psi+4 i \frac{\partial \psi}{\partial \zeta}=0$, where $\varepsilon=w_{0} / z_{R}$ and $\nabla_{\perp}^{2}=$ $\frac{\partial^{2}}{\partial \xi^{2}}+\frac{\partial^{2}}{\partial \eta^{2}}$, with $w_{0}$ the waist of the laser beam and $z_{R}$ its Rayleigh length. The solution of this equation can be obtained by means of a perturbation method[8] when $\varepsilon$ is sufficiently small. It reads

$$
\psi=\sum_{n=0}^{\infty} \varepsilon^{2 n} \psi_{2 n} .
$$

Successive orders $\psi_{n \geq 1}$ are solved from the recursive equations by equalizing the terms with the same order of $\varepsilon^{2}$

$$
\left(\nabla_{\perp}^{2}+4 i \frac{\partial}{\partial \zeta}\right) \psi_{2 n}= \begin{cases}0, & \text { if } n=0 \\ -\frac{\partial^{2} \psi_{2(n-1)}}{\partial \zeta^{2}}, & \text { if } n>0 .\end{cases}
$$

The $n=0$ paraxial solution is $\psi_{0}=f \exp \left(-f \rho^{2}\right)$ where $f=1 /(1+i \zeta)$. Solutions of the higher order can be obtained by solving recursively in momentum space the Eq. (4), that translates into

$$
\left(-\kappa_{\tilde{\zeta}}^{2}-\kappa_{\eta}^{2}+4 i \frac{\partial}{\partial \zeta}\right) \tilde{\psi}_{2 n}= \begin{cases}0, & \text { if } n=0 \\ -\frac{\partial^{2} \tilde{\psi}_{2(n-1)}}{\partial \xi^{2}}, & \text { if } n>0\end{cases}
$$

where $\tilde{\psi}$ is the Fourier transform of $\psi$ and $\kappa_{\xi, \eta}$ are the transverse momenta corresponding to $\xi, \eta$. The paraxial solution of this equation is $\tilde{\psi}_{0}=\exp \left(-\left(\kappa_{\tilde{\xi}}^{2}+\kappa_{\eta}^{2}\right) /(4 f)\right)$. Solutions of these equations can be obtained as $\tilde{\psi}_{2 n}=\tilde{\psi}_{0}\left(Q_{n}+P_{n}\right)$ where $P_{n}$ is a polynomial in $\zeta$ and $\kappa_{\tilde{\xi}}^{2}+\kappa_{\eta}^{2}$ determined by the equation $\tilde{\psi}_{0} P_{n}=-\frac{\partial^{2} \tilde{\psi}_{2(n-1)}}{\partial \zeta^{2}}$ and $Q_{n}=\sum_{j=1}^{n+1-k} \sum_{k=1}^{n+1} C_{2 n, j, k} \kappa_{\xi}^{2(j-1)} \kappa_{\eta}^{2(k-1)}$. It is chosen not to include higher powers of $\kappa_{\tilde{\zeta}, \eta}^{2}$ since they are naturally accounted for by higher order terms of the expansion. The coefficients corresponding to the lower powers of $\kappa_{\xi, \eta}^{2}$ in principle can be set to zero since they correspond to a redefinition of the scaling of the field or of the expansion parameter $\epsilon$. Though we will see they are useful in some cases. In the direct space, the solutions in case of a symmetric $x \leftrightarrow y$ solution of the paraxial expansion of the scalar $\psi$ function can be found in Ref. [9]. It can be useful to consider a more general version where this symmetry is explicitly broken, but yet no general 
astigmatism is introduced [20]. The first term in the expansion reads

$$
\begin{gathered}
\psi_{2}(\xi, \eta, f)=f\left(-\frac{1}{2}+2 C_{2,1,2}+2 C_{2,2,1}\right)+f^{2}\left(\frac{1}{2}+\rho^{2}\right. \\
\left.-4\left(\xi^{2} C_{2,1,2}+\eta^{2} C_{2,2,1}\right)+4 C_{2,2,2}+12\left(C_{2,1,3}+C_{2,3,1}\right)\right) \\
+f^{3}\left(-\frac{\rho^{4}}{4}-\rho^{2}\left(1+8 C_{2,2,2}\right)-48\left(\xi^{2} C_{2,1,3}+\eta^{2} C_{2,3,1}\right)\right) \\
+f^{4}\left(\frac{\rho^{4}}{4}+16\left(\xi^{4} C_{2,1,3}+\eta^{4} C_{2,3,1}\right)+16 \xi^{2} \eta^{2} C_{2,2,2}\right) .
\end{gathered}
$$

These expression match those previously obtained in Ref. [9], where the $x \leftrightarrow y$ is enforced i.e. when $C_{2,1,2}=C_{2,2,1}=\tilde{C}_{2,2}$ and $C_{2,1,3}=C_{2,3,1}=C_{2,2,2} / 2=\tilde{C}_{2,4}$, where the tilde coefficients are those of the Ref. [9]. Higher orders are straightforwardly calculated by means of a symbolic computation software, whose results are piped into a numerical computation software. Obtained expression for the order 2 are too long to be reproduced here but they are similar to those of Ref. [9] with the $x \leftrightarrow y$ relaxed. It is then plugged into Eq. (3), further used in Eq. (2) and to compute the vectors $\vec{A}^{\prime}$ and $\vec{A}^{\prime \prime}$. These $C$ parameters were found to allow matching known solutions of the scalar wave equation expanded up to the second term of the expansion, corresponding to various boundary conditions [9]. They in general depend on the injecting laser beam and the boundary conditions imposed by the optical system and are in principle complex numbers. Indeed nothing bounds them to the real space in the derivation previously described. They can, in principle, be obtained by means of a fit to a given numerical description of the electromagnetic field numerically derived or experimentally measured, up to a certain precision. The procedure that is described here is strictly valid for monochromatic pulses only, which is sufficient in the case studied in this article, though it can be extended to generally astigmatic beams [20] and electromagnetic fields involving spatio-temporal couplings [11]. Extreme cases where a very broad angular spectrum as those considered for instance in [21] are unlikely described by such series expansion, owing to large values of the expansion parameter $\varepsilon$.

\section{IGNATOVSKY FORMALISM}

The Ignatovsky solution[7] is derived from a collimated linearly polarized Gaussian beam $\vec{E}_{i}(x, y, z)=\hat{x} E_{e n v} e^{i\left(k z-\omega_{0} t\right)}$, where $w_{i}$ is the beam radius of the beam and $E_{\text {env }}=E_{0} e^{-\left(x^{2}+y^{2}\right) / w_{i}^{2}}$, focused by a perfect and perfectly aligned parabolic mirror with focal length $f$ whose surface is denoted as $z^{\prime}=-f+\left(x^{\prime 2}+\right.$ $\left.y^{\prime 2}\right) /(4 f)$, in which $\left(x^{\prime}, y^{\prime}, z^{\prime}\right)$ are specialized to represent the points on the parabolic mirror surface. Several approximations are made in the derivation. First of all the Debye approximation is employed, the focusing mirror residing far from the focus, and the transverse electromagnetic field envelope is supposed to vary slowly laterally on the mirror surface. The reflected fields [7] are expressed as

$$
\begin{aligned}
\vec{E}(x, y, z)= & -i k f e^{i\left(k f-\omega_{0} t\right)} \\
& {\left[\hat{x}\left(I_{0}+\frac{x^{2}-y^{2}}{r^{2}} I_{2}\right)+\hat{y} \frac{2 x y}{r^{2}} I_{2}-i \hat{z} \frac{x}{r} I_{1}\right], }
\end{aligned}
$$

$$
\begin{aligned}
\vec{B}(x, y, z)=- & i \frac{k f}{c} e^{i\left(k f-\omega_{0} t\right)} \\
& {\left[\hat{x} \frac{2 x y}{r^{2}} I_{2}+\hat{y}\left(I_{0}-\frac{x^{2}-y^{2}}{r^{2}} I_{2}\right)-i \hat{z} \frac{y}{r} I_{1}\right], }
\end{aligned}
$$

in which

$$
\begin{aligned}
& I_{0}=\int_{0}^{\pi} d \theta E_{\text {env }}\left(r^{\prime}\right) J_{0}(k r \sin \theta) \sin \theta e^{i k z \cos \theta} \\
& I_{1}=2 \int_{0}^{\pi} d \theta E_{\text {env }}\left(r^{\prime}\right) \sqrt{\eta(\theta)} J_{1}(k r \sin \theta) \sin \theta e^{i k z \cos \theta} \\
& I_{2}=\int_{0}^{\pi} d \theta E_{\text {env }}\left(r^{\prime}\right) \eta(\theta) J_{2}(k r \sin \theta) \sin \theta e^{i k z \cos \theta},
\end{aligned}
$$

where $x=r \cos \phi, y=r \sin \phi$ and $\eta(\theta)=(1-\cos \theta) /(1+$ $\cos \theta)$.

This integral solution of the Maxwell equations may sound elegant since it provides expressions based on assumptions that are easier to gauge compared to the expression of the field at the focus point, as done in the series expansion method. If it may appear easier to accept that the input beam envelope can be approximated by a pure paraxial Gaussian beam, it may be more difficult to accept that the key focusing element of a corresponding experimental setup may be a perfect surface, aberration free, and perfectly aligned, which would break the symmetry. This renders the practical utility of such an approach difficult to gauge. These integral formulae have been compared against few existing series expansions without much success [7], since they are corresponding to different boundary conditions. However, using a generalized approach as that described in section 2 , may allow to approximate with enough precision these integral formulae and at the same time allowing for gauging effects as mis-alignments by rotating space coordinates and reducing significantly the computer calculation time.

\section{APPROXIMATING THE IGNATOVSKY FORMULAE WITH A SERIES EXPANSION}

As already mentioned, nothing forbids to extract the arbitrary coefficients of the series expansion by a fit on numerical expressions that are obtained, for instance, by a theoretical integral model. We illustrate this possibility in this section by fitting the first order, except otherwise explicitely stated, expansion coefficients to the numerical field values obtained with Ignatovsky's expressions.

The field amplitude is set to be $E_{0}=1$ that is an unnecessary scale factor for the extraction of the free coefficients. We also use $\lambda=1 \mu \mathrm{m}, w_{i}=0.1 \mathrm{~m}, f=2 w_{i}$. For the fitting process the expressions from Ignatovsky are scaled by the value of the electric field along the $x$ direction at the ideal focus point parameterized by $x=0, y=0, z=0$. In order to allow for a slight mismatch in the total field energy, a scale coefficient is introduced in front of terms independent of the previously introduced $C$ coefficients. $N_{c}=7$ coefficients are thus obtained by matrix inversion since the obtained field expressions are linearly dependent on those. The contribution of each of the six projections of the real parts of the electromagnetic field to each term proportional to a given coefficient is filled in a $6 N_{S} \times N_{c}$ matrix denoted $M$. The $6 N_{S}$ real field projections at the $N_{S}$ sampling points for the expression of Ignatovsky is stored in a vector $F$. The best fit coefficients are thus obtained by

$$
C_{\mathrm{f}}=\left(M^{T} \cdot M\right)^{-1} \cdot M^{T} \cdot F,
$$


where the upperscript ${ }^{T}$ denotes the transposition operator. The quality of the fit is gauged by a parameter

$$
\chi^{2}=\sum_{N_{s}}\left|F-M \cdot C_{\mathrm{f}}\right|^{2}
$$

The waist $w_{0}$ of the Gaussian beam used for the series expansion is fixed in this procedure, that is thus repeated for various $w_{0}$ values that are scanned in an appropriate range. The $\chi^{2}$ dependence on the $w_{0}$ value, shown on Fig. 1, is approximately parabolic with a minimum value in the range of interest, thus uniquely defining the best fitting set of coefficients $C_{b}$ associated to a value of $w_{0, b}$ that provide the best approximation of the integral expressions.

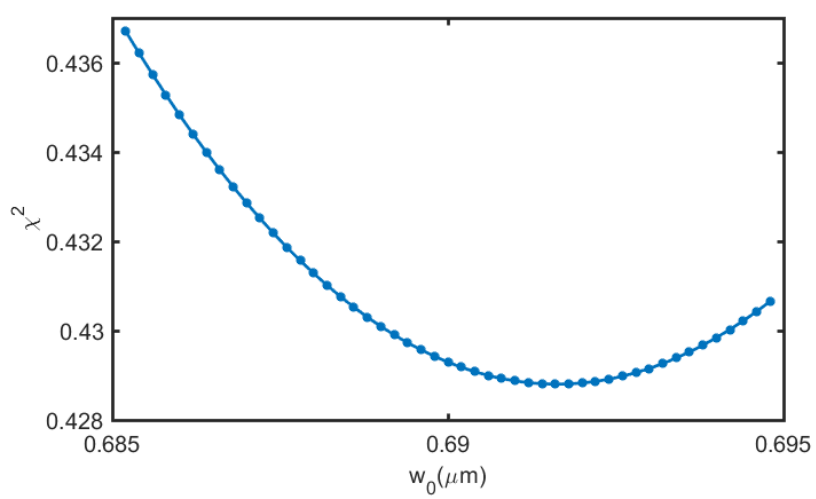

Fig. 1. Example of the dependence of the value of $\chi^{2}$ as function of the value of $w_{0}$ used to define the Gaussian beam that is series expanded according to the technique described in Section 2 . The step size in $w_{0}$ goes down to $0.2 \mathrm{~nm}$. The sampling procedure is the number 2 .

One key ingredient in this procedure consist in the choice of a definite set of sampling points. We decide to always sample randomly the focal plan defined by $z=0$. The choice of the randomization range and distribution is however extremely dependent on the application that is sought for. For instance, in the specific case of direct laser acceleration discussed in the introduction, it is difficult to decide a priori what sampling distribution is most appropriate i.e. the one that allows extracting the set of parameters for which, for instance, the beam energy versus initial position matches best the numerical results based on the numerical integrals from Ignatovsky formulation. Thus we have decided to treat the choice of the sampling distribution as a nuisance, and thus a systematic uncertainty on the extraction of the coefficients. To estimate this effect, five sampling methods are employed. They are listed below:

1. sampling points are Gaussian random distributed with $\sigma_{x, y}=0.5 w_{0 T}$,

2. sampling points are Gaussian random distributed with $\sigma_{x, y}=w_{0 T}$

3. sampling points are uniformly random distributed with $x, y$ ranging within $(0, \lambda)$,

4. sampling points are uniformly random distributed with $x, y$ ranging within $(0,3 \lambda)$, where

$$
w_{0 T}=\sqrt{\frac{w_{i}^{2}-\sqrt{w_{i}^{4}-4 \lambda^{2} f^{2} / \pi^{2}}}{2}}=0.63 \mu \mathrm{m}
$$

is the estimated waist of the paraxial beam. and all the sampling points of either method are taken on the focal plane. Alternatively to sample points in the plane $z=0$ they can also be distributed longitudinally. A uniform longitudinal distribution of points along the $z$ axis in the range $\left[0, z_{R}\right]$ has been investigated along with a transverse distribution of points corresponding to that of sampling number 2. It is found to give a result that lies in between those of samplings 2 and 4 . The improvement is not significant.

The $N_{c}$ parameters calculated with these four sampling methods and the related waist sizes are listed in Table 1. Several remarks are in order. First of all, the $x \leftrightarrow y$ symmetry seem approximately respected, which is expected for a perfectly aligned optical system. The scaling factor $C_{1}$ does not change by more than $5 \%$ and the best fitted waist is stable within $1 \%$ and relatively close to the naive expectation $w_{0 T}$, when considering several sampling distributions. This is somehow expected since these parameters are mainly driven by the overall normalization of the field and the paraxial approximation, respectively. The other parameters are varying more significantly, up to $50 \%$ in relative values. This is expected since their value is driven by the accurateness with which tails of the field distributions are fitted, which as expected depends on the transverse excursion of the sampling points far from the beam center. The obtained parameters do not correspond to values previously obtained [9] as expected from Ref. [7]. In any of the situation the obtained coefficients seem to fit relatively well the Ignatovsky solution. As can be seen on the Fig. 2 for the sampling distribution number 2 , the residuals are within $1 \%$ relative to the maximum field value $E_{x 0}=E_{x}(0,0,0)$. It is apparent that the first diffraction ring that is present in the Ignatovsky model on the $E_{x}$ and $E_{z}$ components is also present in the fitted series expansion though with a different amplitude, contrary to those in the $E_{y}$ component. However second diffraction rings are not present. It is striking that despite the fit is performed in the $z=0$ plane, the obtained parameterization is also matching Ignatosvky model at $z=z_{R}$ within a few per mil of the maximum field value, see Fig. 3. This represents a clear improvement in the approximation of the Ignatovsky model with analytic formulae from series expansions of the paraxial Gaussian beam [7]. As will be seen further the diffraction rings may be of major importance when modeling direct acceleration. We thus investigate the improvements obtained when introducing the next order correction in the series expansion, which adds 9 parameters to the fit. The results are given in Figs. 4 and 5. It is noticed that the description of the tails of the Ignatovsky model improves in the sense that the diffraction rings magnitude are better fitted than with the first order only. A diffraction ring the series expansion model appears in these plots for the $E_{y}$ component which is a new feature with respect to the inclusion of the first order only. The presence of additional diffraction rings when considering higher order terms in the parameterization remains an open point but may well happen. The tails at $z=z_{R}$ are impressively well fitted while the residual of the fit at $\left(x=0, y=0, z=z_{R}\right)$ gets larger when the order 2 is included. 
Table 1. Series expansion parameters that fit best Ignatovsky's model for the four sampling methods. The value of the $\chi^{2}$ is also mentioned.

\begin{tabular}{ccccc}
\hline $\begin{array}{c}\text { sampling } \\
\text { method }\end{array}$ & 1 & 2 & 3 & 4 \\
\hline$C_{1}$ & 0.97 & 0.95 & 0.98 & 0.92 \\
$C_{2,2,1}$ & 0.35 & 0.43 & 0.38 & 0.50 \\
$C_{2,3,1}$ & -0.025 & -0.033 & -0.030 & -0.037 \\
$C_{2,1,2}$ & 0.36 & 0.43 & 0.38 & 0.50 \\
$C_{2,2,2}$ & -0.050 & -0.066 & -0.067 & -0.075 \\
$C_{2,1,3}$ & -0.025 & -0.033 & -0.029 & -0.038 \\
$\omega_{0}(\mu m)$ & 0.69 & 0.69 & 0.68 & 0.69 \\
$\chi^{2}$ & 0.07 & 0.43 & 0.42 & 0.14 \\
\hline
\end{tabular}
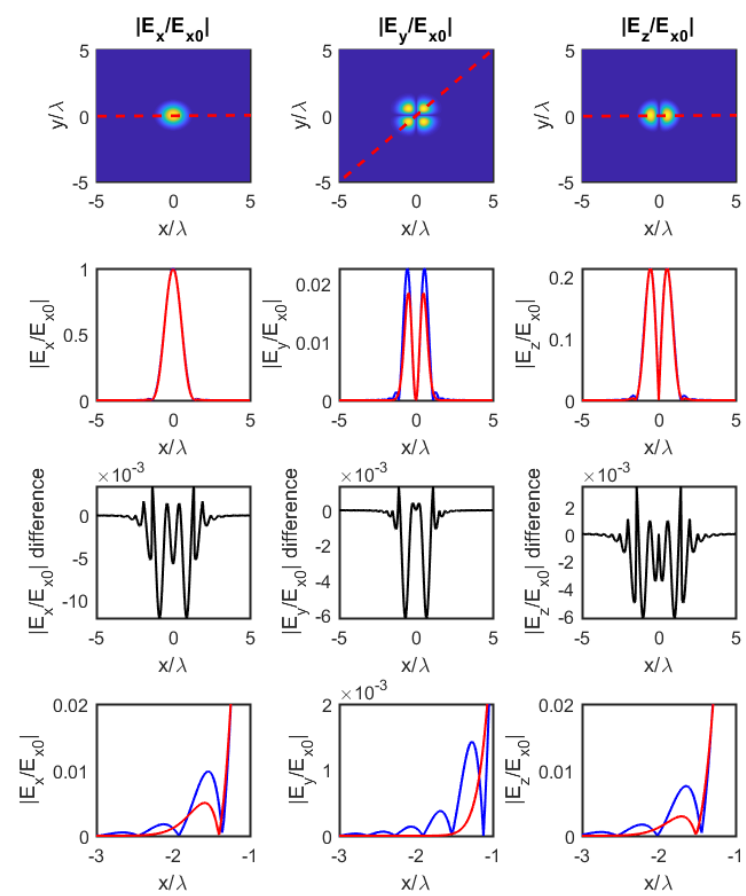

Fig. 2. (Top) magnitude of the complex electric fields components at $z=0$ normalized by its maximum value in the focal plane $E_{x}(0,0,0)$. (Second line) normalized magnitude of the electric field components for the (blue) Ignatovsky model and the (red) generalized series expansion model on the axes that are represented by red dashed lines on the top plots. (Third line) difference between the curves of the middle plots. The differences are in the per mil range which is a clear improvement compared to the results presented in Ref. [7]. (Bottom) zoom on the tails in the region $[-3 \lambda,-\lambda]$ of the normalized magnitude of the electric field components for the (blue) Ignatovsky model and the (red) generalized series expansion model. These plots correspond to the series expansion composed of the first order term in the expansion and the sampling distribution number 2 .
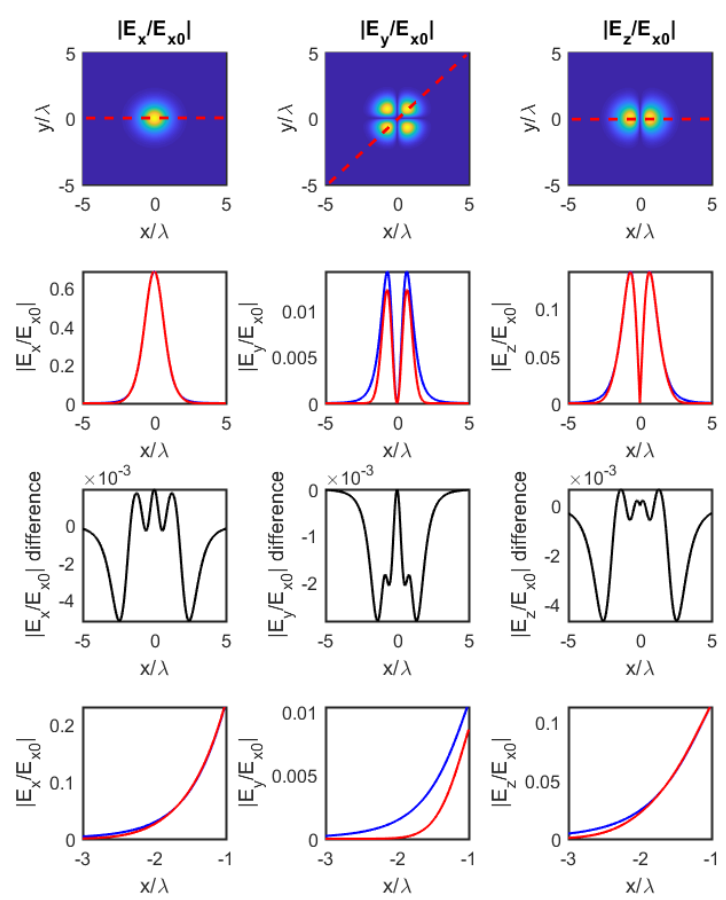

Fig. 3. Same as Fig. 2 except that $z=z_{R}$.
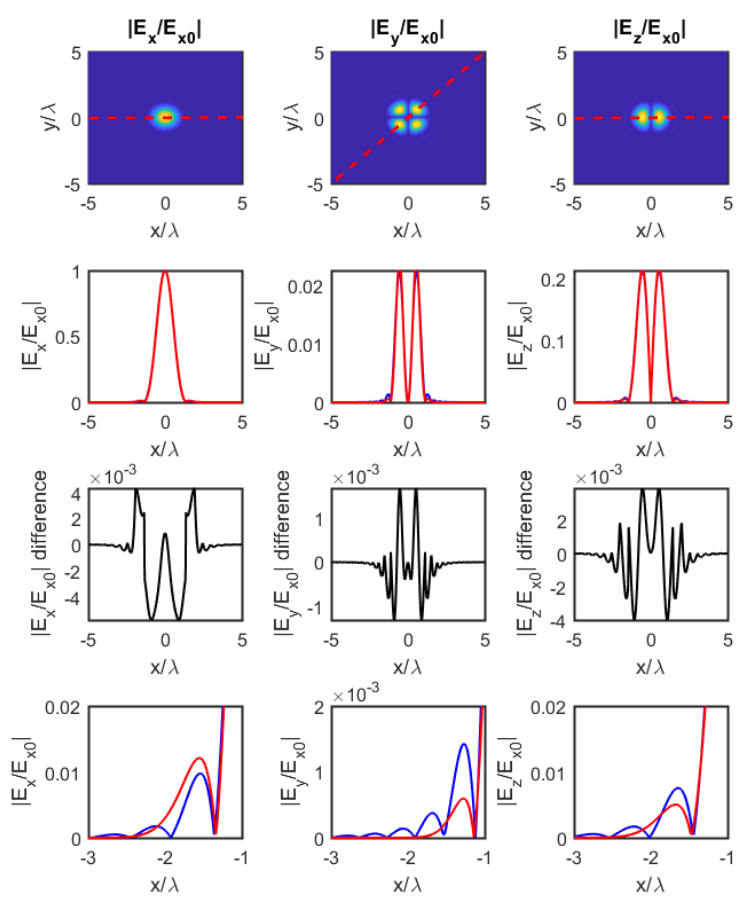

Fig. 4. Same as Fig. 2 except that the series expansion is composed of the first and second order terms in the expansion. 

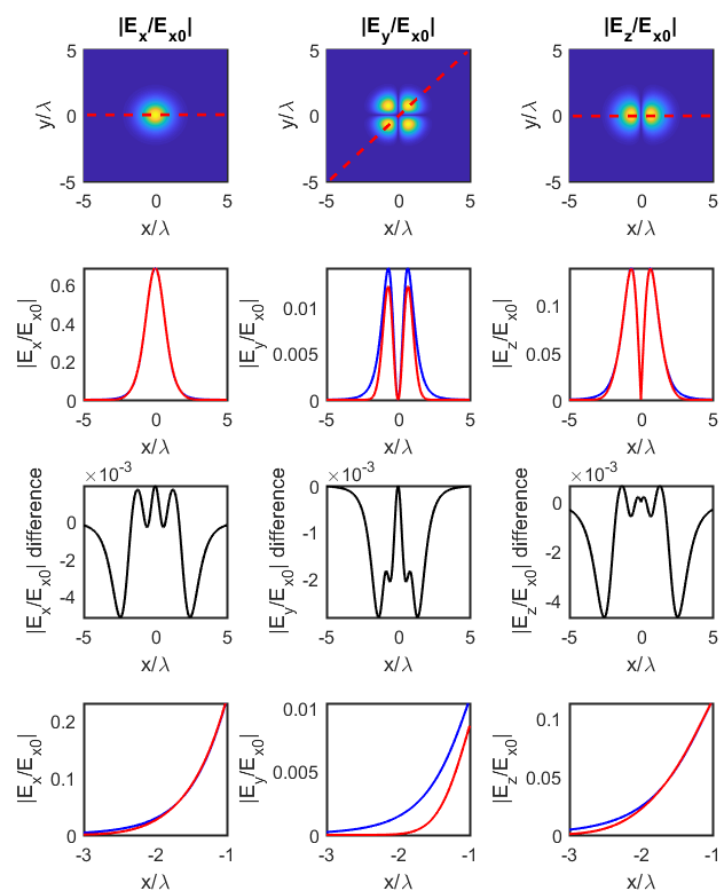

Fig. 5. Same as Fig. 4 except that $z=z_{R}$.

\section{ELECTRON ACCELERATION}

As mentioned in the introduction, we choose to exemplify the usefulness of the series expansion model in the specific case of the acceleration of electrons initially at rest at the focus of the parabola. Such a situation is typical of that of a gas cell placed in this region. Optimization of the acceleration process requires a scan of the parameters space, that is out of the scope of this paper. Indeed we solely intend to assert the effect of the proposed approximations to the Ignatovsky model. To this end we restrict ourselves to a hyperbolic secant longitudinal laser pule $g\left(\phi / \phi_{0}\right)=\operatorname{sech}\left(\phi / \phi_{0}\right)$ with a pulse length $\tau=23 f s$, $\delta \phi=0$ and $P=1 \mathrm{PW}$. The field amplitude is approximated with a first order approximation[22]

$$
E_{0}=\frac{2}{w_{0}} \sqrt{\frac{c \mu_{0}}{\pi} \frac{P}{1+\left(\frac{\varepsilon}{2}\right)^{2}}} .
$$

The effect of this approximation was not found to have a significant impact on the results.

The electrons are assumed to be initially placed at rest at the initial time of the simulation at $y=0$ and $z=0$ but with $x$ varying from 0 to $2 \lambda$. Single electrons acceleration, thus neglecting non-linearities in electron beam dynamics, is calculated by solving Newton-Lorentz equation[23] within a time interval $[-500 \mathrm{fs},+15 \mathrm{ps}]$. Given the illustrative purpose of this section, radiation reaction effects are neglected and would need a careful treatment in the case a predictive simulation is needed[24]. The final energy gain of the electrons accelerated by a pulse modelled with Ignatovsky's, a series expansion with parameters fitted to the Ignatovsky and Erikson's models [25] as a function of the initial transverse position are shown in Fig.6. Erikson's solution was previously found to match best Ignatovsky's model [7] but fails in describing the diffraction rings. It is noticeable that all models give very close results when the electrons are initially located within a transverse position of $x<\lambda$. This can be explained by the fact that, in this region, the driving force comes from the dominant $E_{x}$ field contribution. Electrons are thus pulled away of the $z=0$ axis, drifting progressively along the $x$ axis i.e. towards lower intensity region. It is thus mainly driven by a ponderomotive effect. Because they are initially at rest and are smoothly drifting away from the pulse center, the electrons suffer a large number of optical periods and, in turn, the contribution to the acceleration related to the $E_{z}$ field component is averaged over several cycles. Thus they hardly reach relativistic velocities, except in a very small region nearby the $z$ axis. In this very narrow region the ponderomotive force is also driving the acceleration process. In particular, the dominant contribution is due to the very large gradient of the $E_{z}$ component towards $z=0$ that induces a confinement of the electrons on axis and also a significant net acceleration.

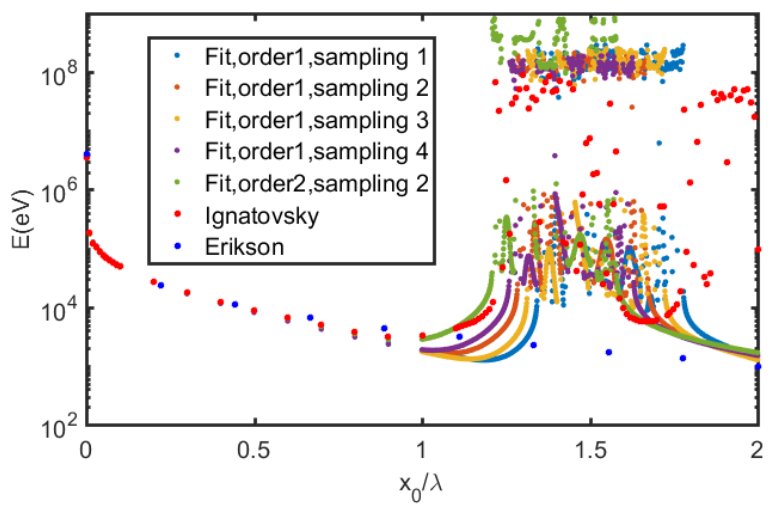

Fig. 6. Net energy gain of electrons after the passage of the laser pulse versus their initial position in $x$. The electrons are initially placed at rest at $z=0, y=0$ and $x \in[0,2 \lambda]$.

Further away $x>1 \lambda$ from the axis, the situation differs since both $E_{x}$ and $E_{z}$ components have a similar magnitude. As can be seen in the Fig. 7 the motion starts to be driven by a ponderomotive effect, as shown by the slow drift of the electron initially at $x=1.5 \lambda$ toward the $z=0$ axis. At a certain point the electron end up in a position in space at an instant when it encounters a sufficiently large $E_{z}$ field contribution, due to the diffraction rings, that induces a large net acceleration over a very small number of cycles. The net acceleration thus depends on tiny details of the history of the electron in the electromagnetic field. In particular the actual number of cycles seen by the acceleration when it starts to accelerate seems to play an important role to determine its final energy. This process is thus extremely dependent on the initial conditions of the problem namely the initial position of the electron but also the exact values of the integration constants of the series expansion model, attested by, for instance, the close fit results and still different net acceleration versus initial transverse position, for sampling 2 and 3. This interpretation is also supported by the fact that Erikson's formulation does not exhibit diffraction rings, and thus this acceleration process is totally nonexistent. Another supporting fact for this interpretation is that acceleration with laser pulses described by Ignatovsky's model exhibit two distinct regions $\left(x_{0} \in[\lambda, 1.75 \lambda]\right.$ and $\left.x_{0}>1.75 \lambda\right)$ where a net acceleration is observed in Fig. 6, due to several diffraction rings visible in, for instance, Fig.2. Finally to this interpretation can be checked 
by looking that the actual $E_{z}$ component of the real part of the electric field at times ranging from $t=-40 \mathrm{fs}$ to $t=-10 \mathrm{fs}$, see Fig.8. It shows that indeed at times when the acceleration takes place for the Ignatovsky's formulations and the series expansion, as determined on Fig. 7, the electron indeed lies in a diffraction ring where this component of the field is large and that it is captured and accelerated significantly. We have checked that when reducing by two orders of magnitude the laser pulse power, this acceleration process in the region $x>\lambda$ does not occur. Indeed in that case the amplitude of the $\mathrm{z}$-component of the electric field is too small to induce a net acceleration. This is also the case when the focal length is increased by a factor two which induces a large reduction of the diffraction rings, that are almost disappearing.
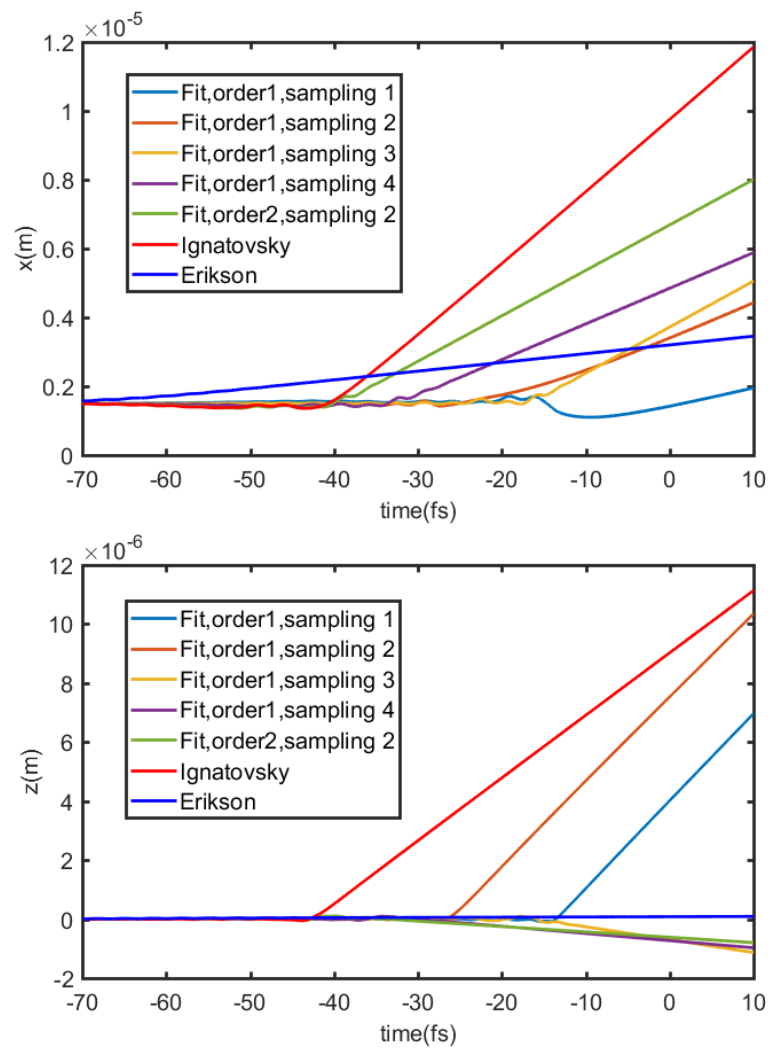

Fig. 7. Trajectories of electrons with initial position $x=$ $1.5 \lambda, y=0, z=0$ in a time range of $-70 \mathrm{fs}$ to $+10 \mathrm{fs}$ for the various models of laser pulses discussed in the text.

This effect could be used for direct laser electron acceleration. Indeed electrons are accelerated up to several tens of $\mathrm{MeV}$, see Fig. 9. The beam energy and energy spread of actually significantly accelerated electrons, thus escaping the interaction region, are similar despite the sensitivity to initial conditions. However they are emitted in a cone that as a large of about one radian which makes them in practice extremely difficult to collect. The simple case studied here is also not much representative of the reality since electrons would have more spread initial positions and velocities. In order to also overcome the fact that a majority of electrons are backscattered to the focusing parabola, acceleration of relativistic electrons produced by other means could be envisaged, for instance exploiting radially polarized beams, provided that the initial beam position, injection time and angular direction is optimized. This study is out of the scope of
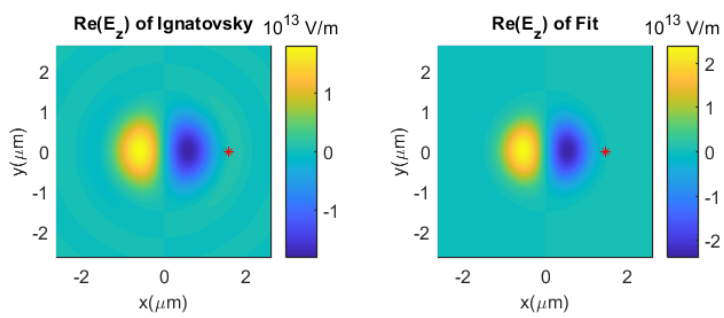

$\operatorname{Re}\left(\mathrm{E}_{\mathbf{z}}\right)$ of Ignatovsky $10^{13} \mathrm{~V} / \mathrm{m}$
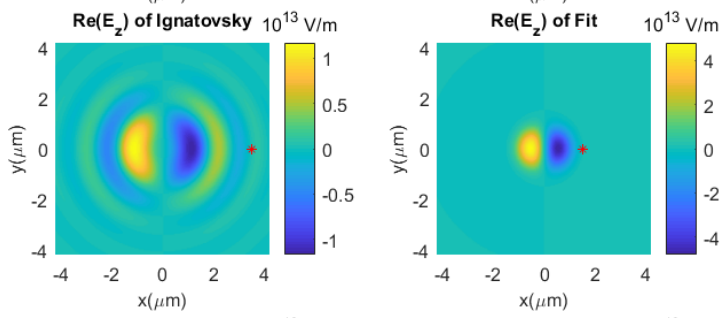

$\operatorname{Re}\left(E_{z}\right)$ of Ignatovsky $10^{13} \mathrm{~V} / \mathrm{m}$
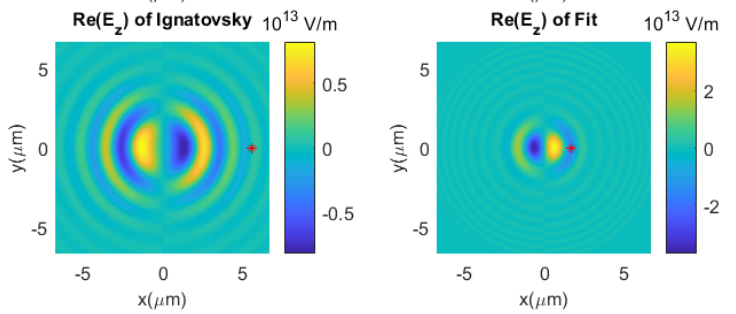

$\operatorname{Re}\left(E_{z}\right)$ of Ignatovsky $10^{13} \mathrm{~V} / \mathrm{m}$
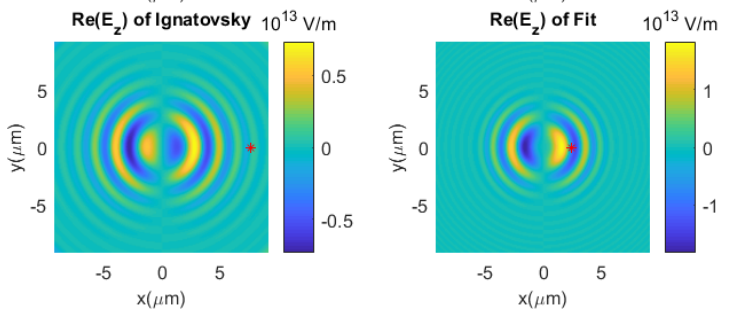

Fig. 8. Electron position (red asterisk) and real part of the $E_{z}$ component of the electromagnetic field at (top) $t=-40 \mathrm{fs}$, $-30 \mathrm{fs},-20 \mathrm{fs}$ and (bottom) -10 fs for the laser pulse modelled with (left) Ignatovsky's formulation and (right) the first order fitted series expansion model on the sampling distribution number 2 . The electron is initially at rest at $x=1.5 \lambda, y=0, z=0$.

this paper, but could still provide a way to boost electron beams. Nevertheless this solution could be viable only if it is reproductive, which is doubtful given the extreme sensitivity to the initial conditions that is demonstrated here. As a closing remark, this high sensitivity comes from the extreme focusing studied here. Thus the predictive power of simulations, which is crucial when optimising an experimental setup, with this acceleration scheme is expected to improve significantly by avoiding geometries with extreme focusing as the one studied here.

\section{SUMMARY}

In this paper, after introducing the necessary formalism, we have shown that it is possible to use a generalized Lax series expansion beyond the paraxial approximation to model an integral formulation of the tight focusing of an electromagnetic field by a parabola. Arbitrary integration constants are introduced in the series expansion and numerically fit to the expression of the electromagnetic field computed using the integrals derived by Ignatovsky. These coefficients were shown to depend on the range over which they are fit. Both formulations were then 


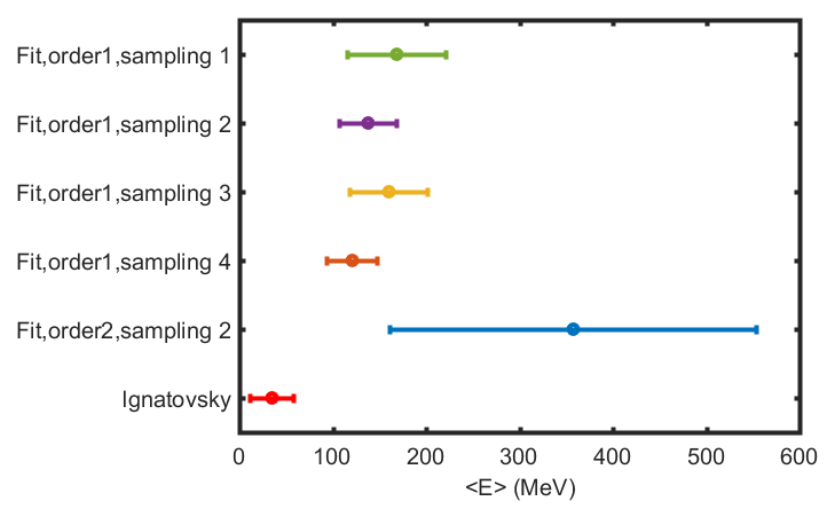

Fig. 9. Average (dots) and standard deviation (error bars) of the energy of electrons that are effectively accelerated with more than $1 \mathrm{MeV}$ net energy gain, for a laser pulse modelled with the Ignatovsky formulae or with the series expansion for various distributions of sampling points.

benchmarked against a simplified direct acceleration scheme. It is found that the various formulations provide significantly different net accelerations depending on the initial conditions. Tight focusing is also found to be the source of an extreme sensitivity to the initial conditions, that jeopardizes the predictive power of such simulations. Indeed this process is sensitive to tiny details in the description of the electromagnetic fields.

\section{REFERENCES}

1. S. Payeur, S. Fourmaux, B. E. Schmidt, J. P. MacLean, C. Tchervenkov, F. Légaré, M. Piché, and J. C. Kieffer, "Generation of a beam of fast electrons by tightly focusing a radially polarized ultrashort laser pulse," Appl. Phys. Lett. 101, 041105 (2012).

2. V. Marceau, P. Hogan-Lamarre, T. Brabec, M. Piché, and C. Varin, "Tunable high-repetition-rate femtosecond few-hundred keV electron source," J. Phys. B: At. Mol. Opt. Phys. 48, 045601 (2015).

3. M. Thévenet, A. Leblanc, S. Kahaly, H. Vincenti, A. Vernier, F. Quéré, and J. Faure, "Vacuum laser acceleration of relativistic electrons using plasma mirror injectors," Nat. Phys. 12, 355-360 (2015).

4. N. D. Powers, I. Ghebregziabher, G. Golovin, C. Liu, S. Chen, S. Banerjee, J. Zhang, and D. P. Umstadter, "Quasi-monoenergetic and tunable $\mathrm{X}$-rays from a laser-driven compton light source," Nat. Photonics 8, 28-31 (2014).

5. P. L. Fortin, M. Piché, and C. Varin, "Direct-field electron acceleration with ultrafast radially polarized laser beams: scaling laws and optimization," J. Phys. B: At. Mol. Opt. Phys. 43, 025401 (2009).

6. A. April, "Nonparaxial TM and TE beams in free space," Opt. Lett. 33, 1563-1565 (2008).

7. J. Peatross, M. Berrondo, D. Smith, and M. Ware, "Vector fields in a tight laser focus: comparison of models," Opt. Express 25, 13990 (2017).

8. M. Lax, W. H. Louisell, and W. B. McKnight, "From Maxwell to paraxial wave optics," Phys. Rev. A 11, 1365-1370 (1975).

9. A. Martens, K. Dupraz, K. Cassou, N. Delerue, A. Variola, and F. Zomer, "Direct electron acceleration with tightly focused $\mathrm{TM}_{0,1}$ beams: boundary conditions and non-paraxial corrections," Opt. Lett. 39, 981-984 (2014).

10. S. W. Jolly, "Influence of longitudinal chromatism on vacuum acceleration by intense radially polarized laser beams," Opt. Lett. 44, 1833 (2019).

11. P. Favier, K. Dupraz, K. Cassou, X. Liu, A. Martens, C. F. Ndiaye, T. Williams, and F. Zomer, "Short pulse laser beam beyond paraxial approximation," J. Opt. Soc. Am. A 34, 1351 (2017).

12. P. Gonález de Alaiza Martínez, G. Duchateau, B. Chimier, R. Nuter,
I. Thiele, S. Skupin, and V. T. Tikhonchuk, "Maxwell-consistent, symmetry- and energy-preserving solutions for ultrashort-laser-pulse propagation beyond the paraxial approximation," Phys. Rev. A 98, 043849 (2018).

13. L. Dai, J.-X. Li, W.-P. Zang, and T. Jian-Guo, "Vacuum electron acceleration driven by a tightly focused radially polarized gaussian beam," Opt. Expresses 19, 9303-9308 (2011).

14. R. Borghi and M. Santarsiero, "Summing Lax series for nonparaxial beam propogation," Opt. Lett. 28, 774-776 (2003).

15. V. S. Ignatovsky, "Diffraction by a parabolic mirror having arbitrary opening," Transactions Opt. Inst. Petrograd 1, 5 (1920).

16. L. W. Davis, "Theory of electromagnetic beams," Phys. Rev. A 19, 1177-1179 (1979).

17. J. P. Barton and D. R. Alexander, "Fifth-order corrected electromagnetic field components for a fundamental gaussian beam," J. Appl. Phys. 66, 2800-2802 (1989).

18. A. L. Cullen and P. K. Yu, "Complex source-point theory of the electromagnetic open resonator," Proc. Royal Soc. Lond. A. Math. Phys. Sci. 366, 155-171 (1979).

19. A. April, "Ultrashort, Strongly Focused Laser Pulses in Free Space," in Coherence and Ultrashort Pulse Laser Emission, F. J. Duarte, ed. (InTech, 2010).

20. X. Liu, K. Cassou, K. Dupraz, P. Favier, W. H. Huang, A. Martens, C. F. N'Diaye, C. X. Tang, T. Williams, L. X. Yan, and F. Zomer, "S-shaped non-paraxial corrections to general astigmatic beams," J. Opt. Soc. Am. A 34, 576-582 (2017).

21. F. Maucher, S. Skupin, S. A. Gardiner, and I. G. Hughes, "Creating complex optical longitudinal polarization structures," Phys. Rev. Lett. 120, 163903 (2018).

22. Y. Salamin, "Fields of a Gaussian beam beyond the paraxial approximation," Appl. Phys. B 86, 319-326 (2007).

23. V. Marceau, C. Varin, and M. Piché, "Validity of the paraxial approximation for electron acceleration with radially polarized laser beams," Opt. Lett. 38, 821 (2013).

24. C. N. Harvey, A. Gonoskov, A. Ilderton, and M. Marklund, "Quantum quenching of radiation losses in short laser pulses," Phys. Rev. Lett. 118, 105004 (2017).

25. W. Erikson and S. Singh, "Polarization properties of Maxwell-Gaussian laser beams," Phys. Rev. E 49, 5778-5786 (1994). 